\title{
Retrospective validation of 11-13 weeks' gestation ultrasound characteristics as predictive tools for twin-twin transfusion syndrome and selective intrauterine growth restriction in monochorionic diamniotic twin pregnancies
}

\author{
Yin-Di Zhu ${ }^{1 \#}$, Jin-Yan Bian ${ }^{1 \#}$, Yu-Ping Liao ${ }^{2 \#}$, Ting Hu ${ }^{1}$, Ming-Yue Wang ${ }^{1}$, You-Guo Chen ${ }^{1}$, \\ Mei-Fang Pan $^{3}$, Xin-Xian Gu ${ }^{1,4}$ \\ ${ }^{1}$ Department of Gynecology and Obstetrics, The First Affiliated Hospital of Soochow University, Suzhou, China; ${ }^{2}$ Department of Cardiovascular \\ Medicine, The First Affiliated Hospital of Soochow University, Suzhou, China; ${ }^{3}$ Department of Ultrasound, Suzhou Xiangcheng People's Hospital, \\ Suzhou, China; ${ }^{4}$ Department of Ultrasound, Dushu Lake Hospital affiliated to Soochow University, Suzhou, China \\ Contributions: (I) Conception and design: YD Zhu, JY Bian; (II) Administrative support: XX Gu; (III) Provision of study materials or patients: YP \\ Liao, MF Pan; (IV) Collection and assembly of data: T Hu, YG Chen; (V) Data analysis and interpretation: T Hu, MY Wang; (VI) Manuscript \\ writing: All authors; (VII) Final approval of manuscript: All authors. \\ "These authors contributed equally to this work. \\ Correspondence to: Xin-Xian Gu. Department of Ultrasound, Dushu Lake Hospital Affiliated to Soochow University, Suzhou 215124, China. Email: \\ guxinxian@suda.edu.cn; You-Guo Chen. Department of Gynecology and Obstetrics, The First Hospital Affiliated of Soochow University, Suzhou \\ 215006, China. Email: chenyouguo@suda.edu.cn; Mei-Fang Pan. Department of Ultrasound, Suzhou Xiangcheng People’s Hospital, Suzhou 215131, \\ China. Email: pmf2004@126.com.
}

Background: Twin to twin transfusion syndrome (TTTS) is a serious syndrome that can affect twin pregnancies involving a single placenta, impacts some of twin gestations with monochorionic diamniotic (MCDA) placentas. We validated the ultrasound characteristics of 11-13 weeks' gestation to predict TTTS and selective intrauterine growth restriction (sIUGR) in MCDA pregnancies.

Methods: We retrospectively included all of the MCDA twin pregnancies with ultrasound characteristics, including the crown-rump length (CRL), ductus venosus pulsatility index for veins (DV PIV), and nuchal translucency (NT) thickness, at 11-13 weeks' gestation, followed by mean difference and discordance comparison. Receiver operating characteristic (ROC) curves were constructed for the comparison of values of these predictive markers for identification of MCDA pregnancies with high-risk of adverse outcomes.

Results: A total of 98 MCDA pregnancies were included in this study. Among the 98, 34 (34.7\%) developed sIUGR, whereas 10 (10.2\%) expressed TTTS. Significant differences in NT discordance were found among the normal, sIUGR, and TTTS groups; moreover, a significant difference was found between pregnancies with normal outcomes and sIUGR $(\mathrm{P}<0.001)$, normal and TTTS $(\mathrm{P}<0.001)$, and sIUGR and TTTS $(\mathrm{P}<0.001)$. Difference in NT was determined to be the best predictive marker for sIUGR [area under the curve $(\mathrm{AUC})=0.769 ; 95 \%$ confidence interval (CI): 0.591 to 0.992 ], and NT discordance was considered the best predictive marker for TTTS (AUC $=0.802$; $95 \%$ CI: 0.485 to 0.936 ).

Conclusions: Significant differences in NT discordance were found between the normal, sIUGR, and TTTS groups, while NT difference and NT discordance were identified as predictive markers for sIUGR and TTTS, respectively.

Keywords: Monochorionic diamniotic (MCDA); twin to twin transfusion syndrome (TTTS); selective intrauterine growth restriction (sIUGR)

Submitted Jun 14, 2021. Accepted for publication Sep 10, 2021.

doi: 10.21037/atm-21-3826

View this article at: https://dx.doi.org/10.21037/atm-21-3826 


\section{Introduction}

Monochorionic diamniotic (MCDA) twins account for $70 \%$ of monozygotic (MZ) twins that result from division between 4 to 8 days after fertilization (1-3), and are associated with increased perinatal mortality and morbidity resulting from shared placenta (4-6) and the presence of inter-twin placental vascular anastomoses (7). Twin to twin transfusion syndrome (TTTS) and selective intrauterine growth restriction (sIUGR) are the 2 main risks of MDCA, with prevalence rates of $15-20 \%$ and $15 \%$, respectively $(3,8)$. Due to the higher prevalence of TTTS and sIUGR, early prediction is necessary for effective surveillance. A number of studies have shown that at $11-13+6$ weeks of pregnancy, abnormal crown-to-ruminal length (CRL), cervical translucency (NT), and venous catheter (DV) waveforms are observed in pregnancies with monochorionic complications (9-13). However, most of the studies included a small number of participants and only used the mean difference data of CRL, NT, and DV. The purpose of this study was to use the mean difference and discordance data of CRL, NT, and DV to verify the ultrasound features of pregnancy at 11-13 weeks as a predictive tool for TTTS and sIUGR in MCDA pregnancy.

We present the following article in accordance with the STARD reporting checklist (available at https://dx.doi. org/10.21037/atm-21-3826).

\section{Methods}

This was a retrospective cohort study carried out using data from The First Affiliated Hospital of Soochow University from between January 2015 and December 2020. Women with MCDA twin pregnancies at 11-13+6 weeks of gestation were included in this study. The diagnostic criteria were based on the presence of a single placental mass and the absence of a previously described bimodal sign (14). Pregnancies with severe structural abnormalities in 1 or both twins, including chromosomal abnormalities, loss of 1 or both twins or histological chorionic placental changes were precluded. We evaluated 6 potential characteristics of MCDA complications, including differences and discordance in the CRL, NT thicknesses, and DV pulsatility index for veins (PIV), respectively. All included MCDA twins had known pregnancy outcomes. The NT and CRL were measured by ultrasound at 11-13+6 weeks of gestation. The DV PIV were measured according to standard techniques (15).
Discordances among CRL, NT, and DV PIV in MCDA twin pairs were calculated as the percentage of the larger twin. Pregnancy outcome data were drawn from ultrasound examination data, obstetric electronic medical records, or telephone follow-up. The sIUGR was defined as either a prenatal description based on an estimated difference in fetal weight: a $20 \%$ discordance in the measurements leading to prenatal intervention, or as a difference in birth weight of less than $10 \mathrm{~g}$ between a pair of twins (4).

All procedures performed in this study involving human participants were in accordance with the Declaration of Helsinki (as revised in 2013). The study was approved by the Ethics Committee of The First Affiliated Hospital of Soochow University (No. 21-011). Because of the retrospective nature of the research, the requirement for informed consent was waived.

\section{Statistical analysis}

The statistical analysis and construction of figures were performed using R software version 4.03 (http://www. R-project.org/). The Wilcoxon test and Kruskal-Wallis test were used to compare the discrepancies in twin characteristics (CRL, NT, and DV PIV) in different pregnancy outcome groups between single groups and multiple groups, respectively. The diagnostic values of the above markers in identification of high-risk cohorts at risk of adverse pregnancy outcomes were evaluated using receiver operating characteristic (ROC) curves using "pROC" package in R. Optimal cut-off was calculated using Youden index. A P value $<0.05$ was considered to have statistical significance.

\section{Results}

\section{The maternal and fetal characteristics of the MCDA twin pregnancies cobort}

A total of 98 MDCA pregnancies were included in present study. The maternal and fetal characteristics of the MCDA twin pregnancies cohort are listed in Table 1. A total of 10 cases $(10.2 \%)$ were diagnosed with TTTS at an average gestational age of 18 weeks. There were 6 women with stage 2-3 TTTS who chose to reduce their amniotic fluid, resulting in 3 twin survivors and 3 singleton survivors, and 4 women with stage 3 disease at 20 weeks gestation who chose to terminate their pregnancy. There was sIUGR detected in $34(34.7 \%)$ pregnancies. A total of 2 patients chose to 
Table 1 Maternal and fetal characteristics of the MCDA twin pregnancies cohort $(\mathrm{n}=98)$

\begin{tabular}{lc}
\hline Characteristics & Data \\
\hline Maternal age (years, range) & $24-40$ \\
Median maternal age (years) & 33 \\
Mean gestational age at recruitment (weeks) & $11+4$ \\
Method of conception (n, \%) & $19(19.4)$ \\
Natural & $28(28.6)$ \\
Assisted conception (In-vitro fertilization) & $51(52.0)$ \\
Assisted conception (Ovulation induction drugs) & \\
Maternal fertility history (n, \%) & $77(78.6)$ \\
Nulliparity & $21(21.4)$ \\
Parous & \\
Outcome (n, \%) & $54(55.1)$ \\
Normal & $34(34.7)$ \\
sIUGR & $10(10.2)$ \\
TTTS &
\end{tabular}

MCDA, monochorionic diamniotic; sIUGR, selective intrauterine growth restriction; TTS, twin to twin transfusion syndrome.

terminate their pregnancy due to umbilical cord occlusion at 18 and 19 weeks, respectively. The surviving twins had to be delivered at 32 weeks because they also had IUGR. In 1 of the pregnancies, a small twin died at 21 weeks, and identical twins were delivered at 35 weeks of another pregnancy without obvious neurodevelopmental defects at birth. All remaining sIUGR pregnancies were delivered before 35 weeks.

\section{Comparing the discordance of markers in cohorts with normal pregnancy outcomes and those affected by adverse outcomes}

The comparison of the inconsistencies in markers between pregnancy with normal results and cohorts affected by adverse results is shown in Figure 1. On NT difference, a significant difference was detected between pregnancies with normal outcomes and TTTS $(\mathrm{P}=0.031)$. On NT discordance, significant differences in NT discordance were revealed among the normal, sIUGR, and TTTS groups; moreover, a significant difference was found between pregnancies with normal outcome and sIUGR $(\mathrm{P}<0.001)$, normal and TTTS $(\mathrm{P}<0.001)$, and sIUGR and
TTTS $(\mathrm{P}<0.001)$. Furthermore, on DV PIV difference, a significant difference was found between pregnancies with sIUGR and normal pregnancies $(\mathrm{P}<0.001)$. In addition, no significant difference was found in CRL difference, CRL discordance, and DV PIV discordance.

\section{Diagnostic efficacy of CRL, NT, and DV PIV in prediction of sIUGR and TTTS}

The performance of NT, CRL, and DV PIV as predictive markers of sIUGR is shown in Figure 2. The NT difference was the most effective feature for predicting sIUGR [ROC area under the curve (AUC) $=0.769 ; 95 \% \mathrm{CI}: 0.591$ to 0.992], and other markers from high to low sequence were as follows: DV PIV difference (AUC $=0.698 ; 95 \%$ CI: 0.458 to 0.843 ), NT discordance (AUC $=0.691 ; 95 \%$ CI: 0.425 to 0.786), DV PIV discordance (AUC $=0.564 ; 95 \%$ CI: 0.412 to 0.758 ), CRL discordance (AUC $=0.534 ; 95 \%$ CI: 0.316 to 0.627 ), CRL difference (AUC $=0.503 ; 95 \% \mathrm{CI}: 0.364$ to $0.753)$. In addition, the best cutoff value for NT difference was 0.35 , and the corresponding sensitivity and specificity were 0.766 and 0.765 , respectively. The performance of NT, CRL, and DV PIV as TTTS prediction markers is shown in Figure 3. The NT discordance was the most effective feature for predicting TTTS (ROC AUC $=0.802 ; 95 \%$ CI: 0.485 to 0.936 ), while NT difference (AUC $=0.653 ; 95 \%$ CI: 0.411 to 0.732 ), CRL difference (AUC $=0.564 ; 95 \%$ CI: 0.385 to 0.786 ) and the difference between DV PIV (AUC $=0.553 ; 95 \%$ CI: 0.406 to 0.773 ) showed moderate efficacy. In addition, the inconsistent DV (AUC $=0.502 ; 95 \% \mathrm{CI}$ : 0.382 to 0.721 ) and the inconsistent CRL (AUC $=0.420$; $95 \%$ CI: 0.285 to 0.614 ) were very poor predictive markers. The optimal cutoff value for NT discordance $>20 \%$ was 23.5 , and the corresponding sensitivity and specificity were 0.727 and 0.900 , respectively (Table 2 ).

\section{Discussion}

According to previous studies, the incidence of NT increase/inconsistency in MCDA twins ranges from $8 \%$ to $18 \%$ (9-13). This phenomenon was first described by Sebire et al. in a retrospective cohort of 287 monochorionic twins, and a positive likelihood ratio of 3.5 was obtained, that is, an increase in NT ( $>95$ quantile) for subsequent of TTTS development (9). In our retrospective cohort, NT discordance $>20 \%$ presented in $24.1 \%$ (13/54) of normal pregnancies, $38.2 \%$ (13/34) of sIUGR, and 90\% (9/10) of TTTS. Kagan et al. found that $>20 \%$ of discordance in 

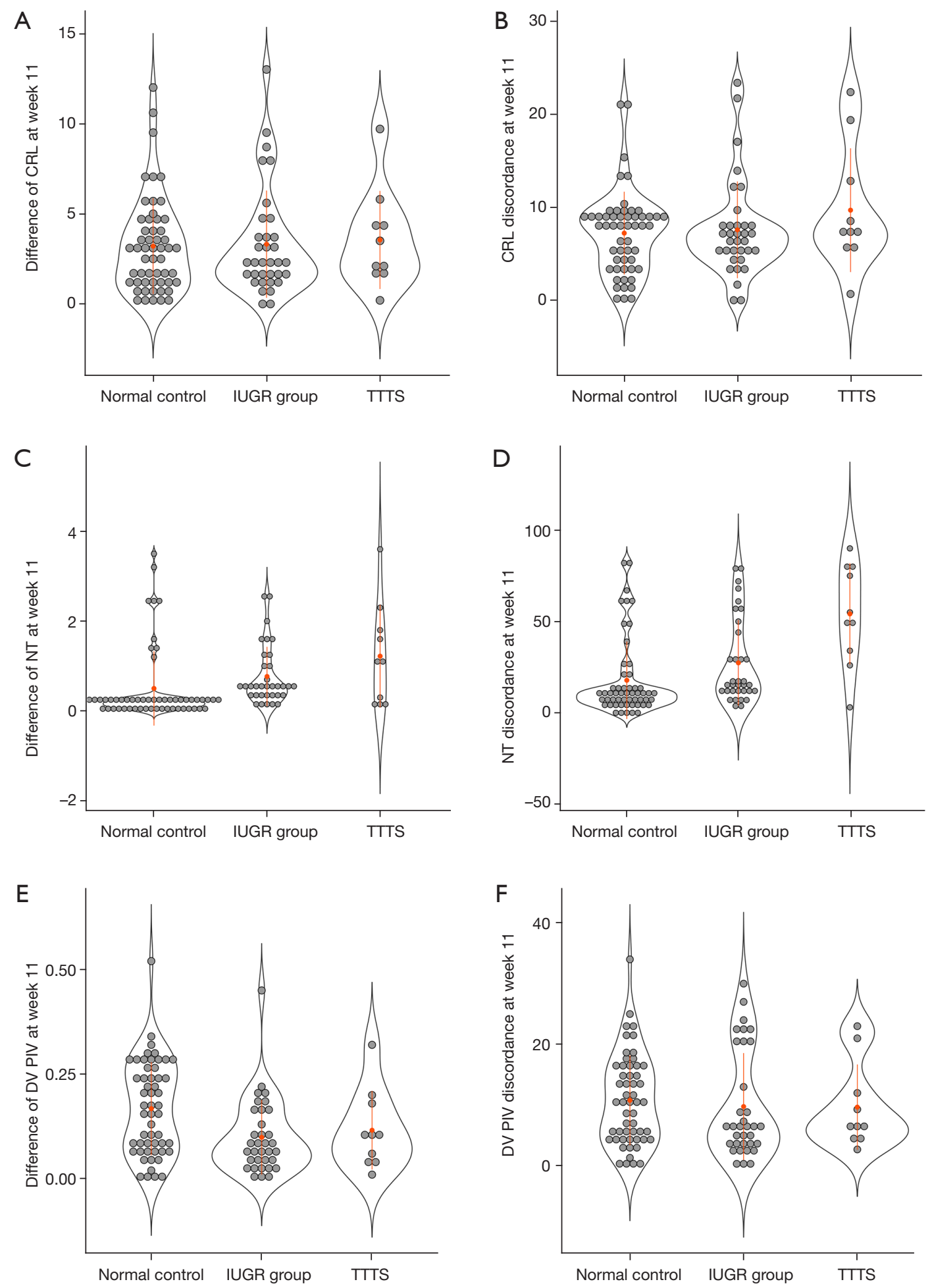

Figure 1 Violin figures were constructed for comparison of the different ultrasound physiological indexes among normal control, IUGR, and TTTS. (A) CRL at week 11; (B) CRL discordance at week 11; (C) difference of NT at week 11; (D) NT discordance at week 11; (E) DV PIV at week 11; (F) DV PIV discordance at week 11. IUGR, intrauterine growth restriction; TTTS, twin to twin transfusion syndrome; CRL, crown to rump length; NT, nuchal translucency; DV PIV, ductus venosus pulsatility index for veins. 


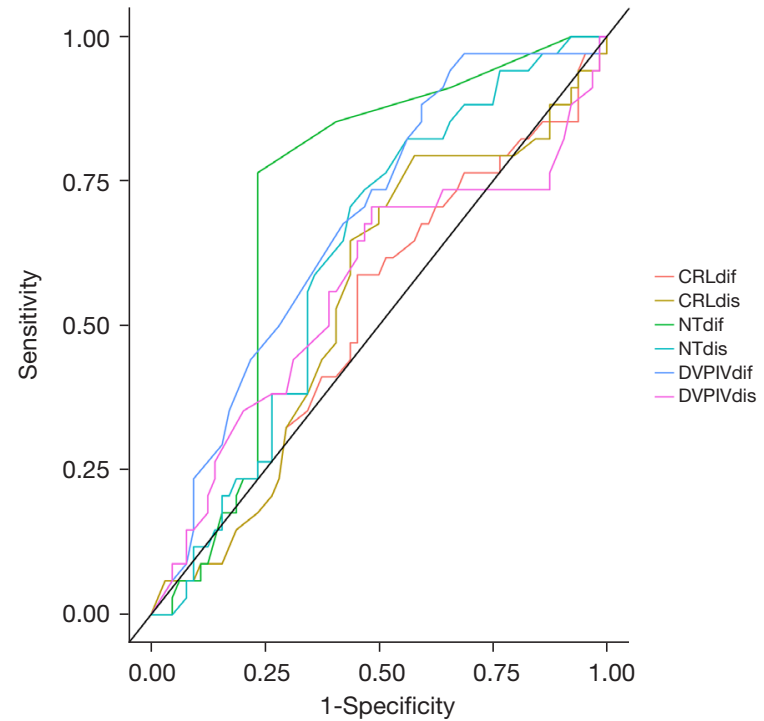

Figure 2 ROC was drawn to illustrate the diagnostic efficacy of different ultrasound indexes for restriction IUGR. ROC, receiver operator curves; IUGR, intrauterine growth restriction.

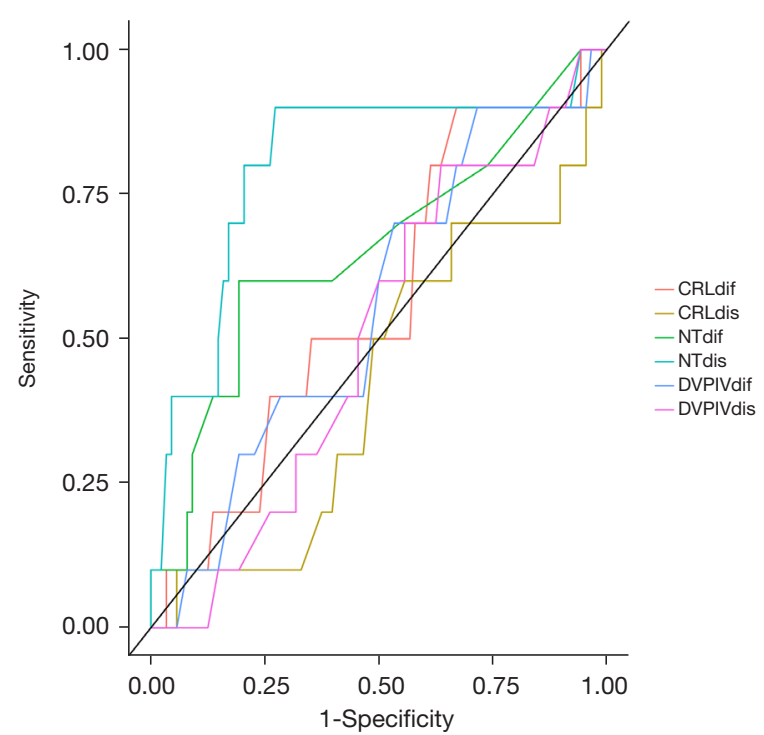

Figure 3 ROC was drawn to illustrate the diagnostic efficacy of different ultrasound indexes for TTTS. ROC, receiver operator curve; TTTS, twin to twin transfusion syndrome.

NT was associated with $50 \%$ of TTTS tests, and the false positive rate was $20 \%$ (10). In our results, the sensitivity and specificity of NT discordance ( $>20 \%)$ were $72.7 \%$ (71/98 cases) and $90.0 \%$ (9/10 cases), respectively.

There was evidence that in the case of sIUGR and TTTS, NT discordance was more pronounced. If a therapy was developed that allowed for the functional separation of choroidal vessels in the first trimester of pregnancy $(16,17)$, an NT discordance $>20 \%$ would be an effective tool for determining which cohort should be offered treatment. However, more than 1 in every 3 pregnancies (13 normal in a total of 35 patient cases) treated would be treated based on false positive results and interventions would need to have minimal surgery-related risks. These data suggest that increased NT in the first trimester could be used as a discriminator for the assignment of MCDA twin pregnancies to high-risk or low-risk models for obstetric care and fetal monitoring. There has been conflicting data about the relationship between MCDA twins' CRL discordance and the subsequent development of TTTS. Similar to the findings of Sebire et al. (18), we did not detect a meaningful relationship between CRL discordance and TTTS. Given that there is an unlikely biological link between TTTS (a hemodynamic problem) and abnormal CRL, this is not surprising. The greater biological rationality is that the significant difference in placental area may be related to the difference in twin growth. However, similar to other authors (19), we did not find a meaningful association between CRL discordance in the first trimester and the development of sIUGR in this study. Since the previous reports have shown that the use of DV for TTTS prediction has a sensitivity of $75 \%$ and a specificity of $92 \%(12,13)$, we analyzed the DV data based on the PIV. Although there were more cases in our series (20-23.5\%) with DV PIV discordance (Table 3), the prevalence of pregnancy with unfavorable outcomes was increased compared to the control group. Wohlmuth et al. reported that in pre-TTTS and early disease, the DV blood flow distribution and the time of waveform events change, reflecting abnormal ventricular filling and circulatory imbalance, and they proposed the use of filling time and velocity-time integral (VTI) as predictive factors. Mutual comparison may allow prediction of the evolution of TTTS in MCDA pregnancy (20). However, despite the higher prevalence of discordant DV PIV in the sIUGR and TTTS groups, the number was too small to draw any conclusions.

There were some limitations to the present study. First, the retrospective nature of the study could have resulted in less reliable access to or unavailability of previous treatment history, and possible selection bias of the case and control participants. Secondly, the relatively small number of TTTS cases could have resulted in reporting bias. Therefore, further prospective studies with a high number of sIUGR and TTTS cases should be performed to confirm the results 
Table 2 Optimal cut-off values and corresponding sensitivity and specificity of the NT difference and NT discordance for sIUGR and TTTS

\begin{tabular}{lccc}
\hline Variable & Optimal cut-off & Sensitivity & Specificity \\
\hline NT difference & 0.35 & 0.766 & 0.765 \\
NT discordance $>20 \%$ & 23.5 & 0.727 & 0.900 \\
\hline
\end{tabular}

sIUGR, selective intrauterine growth restriction; TTS, twin to twin transfusion syndrome; NT, nuchal translucency.

Table 3 Discordance between indexes for normal pregnancies and cohort affected by adverse outcome

\begin{tabular}{lccr}
\hline Variable & Normal controls $(\mathrm{n}=54)$ & slUGR $(\mathrm{n}=34)$ & TTS $(\mathrm{n}=10)$ \\
\hline CRL difference $($ mean, mm) & 3.20 & 3.31 & 3.55 \\
CRL discordance $>10 \%$ & $6 / 54(11.1)$ & $6 / 34(17.6)$ & $3 / 10(30.0)$ \\
NT difference (mean, mm) & 0.50 & 0.76 & 1.22 \\
NT discordance $>20 \%$ & $13 / 54(24.1)$ & $13 / 34(38.2)$ & $9 / 10(90.0)$ \\
DV PIV difference (mean) & 0.167 & 0.099 & 0.116 \\
DV PIV discordance $>20 \%$ & $6 / 54(11.1)$ & $8 / 34(23.5)$ & $2 / 10(20.0)$ \\
\hline
\end{tabular}

sIUGR, selective intrauterine growth restriction; TTTS, twin to twin transfusion syndrome; CRL, crown to rump length; NT, nuchal translucency; DV PIV, ductus venosus pulsatility index for veins.

obtained in this study.

In conclusion, we have demonstrated that significant differences on NT discordance were found among the normal, sIUGR, and TTTS groups, while NT difference and NT discordance were respectively identified as the predictive markers for sIUGR and TTTS.

\section{Acknowledgments}

Funding: This work was supported by Education Department of Jiangsu Province (No. 19KJB320020) and The Science and Technology Project Foundation of Suzhou (No. SYS2020107).

\section{Footnote}

Reporting Checklist: The authors have completed the STARD reporting checklist. Available at https://dx.doi. org/10.21037/atm-21-3826

Data Sharing Statement: Available at https://dx.doi. org/10.21037/atm-21-3826

Conflicts of Interest: All authors have completed the ICMJE uniform disclosure form (available at https://dx.doi. org/10.21037/atm-21-3826). The authors have no conflicts of interest to declare.
Ethical Statement: The authors are accountable for all aspects of the work in ensuring that questions related to the accuracy or integrity of any part of the work are appropriately investigated and resolved. All procedures performed in this study involving human participants were in accordance with the Declaration of Helsinki (as revised in 2013). The study was approved by the Ethics Committee of The First Affiliated Hospital of Soochow University (21011). Because of the retrospective nature of the research, the requirement for informed consent was waived.

Open Access Statement: This is an Open Access article distributed in accordance with the Creative Commons Attribution-NonCommercial-NoDerivs 4.0 International License (CC BY-NC-ND 4.0), which permits the noncommercial replication and distribution of the article with the strict proviso that no changes or edits are made and the original work is properly cited (including links to both the formal publication through the relevant DOI and the license). See: https://creativecommons.org/licenses/by-nc-nd/4.0/.

\section{References}

1. Hack KE, Derks JB, Elias SG, et al. Increased perinatal mortality and morbidity in monochorionic versus dichorionic twin pregnancies: clinical implications of a 
large Dutch cohort study. BJOG 2008;115:58-67.

2. Abusheikha N, Salha O, Sharma V, et al. Monozygotic twinning and IVF/ICSI treatment: a report of 11 cases and review of literature. Hum Reprod Update 2000;6:396-403.

3. Ghalili A, McLennan A, Pedersen L, et al. Outcomes of monochorionic diamniotic twin pregnancies: a comparison of assisted and spontaneous conceptions. Aust N Z J Obstet Gynaecol 2013;53:437-42.

4. Valsky DV, Eixarch E, Martinez JM, et al. Selective intrauterine growth restriction in monochorionic twins: pathophysiology, diagnostic approach and management dilemmas. Semin Fetal Neonatal Med 2010;15:342-8.

5. Fick AL, Feldstein VA, Norton ME, et al. Unequal placental sharing and birth weight discordance in monochorionic diamniotic twins. Am J Obstet Gynecol 2006;195:178-83.

6. Sebire NJ, Snijders RJ, Hughes K, et al. The hidden mortality of monochorionic twin pregnancies. Br J Obstet Gynaecol 1997;104:1203-7.

7. Zipori Y, Reidy K, Gilchrist T, et al. The Outcome of Monochorionic Diamniotic Twins Discordant at 11 to $13+6$ Weeks' Gestation. Twin Res Hum Genet 2016;19:692-6.

8. Oldenburg A, Rode L, Bødker B, et al. Influence of chorionicity on perinatal outcome in a large cohort of Danish twin pregnancies. Ultrasound Obstet Gynecol 2012;39:69-74.

9. Sebire NJ, D'Ercole C, Hughes K, et al. Increased nuchal translucency thickness at 10-14 weeks of gestation as a predictor of severe twin-to-twin transfusion syndrome. Ultrasound Obstet Gynecol 1997;10:86-9.

10. Kagan KO, Gazzoni A, Sepulveda-Gonzalez G, et al. Discordance in nuchal translucency thickness in the prediction of severe twin-to-twin transfusion syndrome. Ultrasound Obstet Gynecol 2007;29:527-32.

11. Fratelli N, Prefumo F, Fichera A, et al. Nuchal translucency thickness and crown rump length discordance for the prediction of outcome in monochorionic

Cite this article as: Zhu YD, Bian JY, Liao YP, Hu T, Wang MY, Chen YG, Pan MF, Gu XX. Retrospective validation of 11-13 weeks' gestation ultrasound characteristics as predictive tools for twin-twin transfusion syndrome and selective intrauterine growth restriction in monochorionic diamniotic twin pregnancies. Ann Transl Med 2021;9(18):1417. doi: $10.21037 /$ atm-21-3826 diamniotic pregnancies. Early Hum Dev 2011;87:27-30.

12. Matias A, Montenegro N, Loureiro T, et al. Screening for twin-twin transfusion syndrome at 11-14 weeks of pregnancy: the key role of ductus venosus blood flow assessment. Ultrasound Obstet Gynecol 2010;35:142-8.

13. Maiz N, Nicolaides KH. Ductus venosus in the first trimester: contribution to screening of chromosomal, cardiac defects and monochorionic twin complications. Fetal Diagn Ther 2010;28:65-71.

14. Huisman TW, Stewart PA, Wladimiroff JW. Ductus venosus blood flow velocity waveforms in the human fetus-a Doppler study. Ultrasound Med Biol 1992;18:33-7.

15. Falcon O, Faiola S, Huggon I, et al. Fetal tricuspid regurgitation at the $11+0$ to $13+6$-week scan: association with chromosomal defects and reproducibility of the method. Ultrasound Obstet Gynecol 2006;27:609-12.

16. Shaw CJ, Civale J, Botting KJ, et al. Noninvasive highintensity focused ultrasound treatment of twin-twin transfusion syndrome: A preliminary in vivo study. Sci Transl Med 2016;8:347ra95.

17. Caloone J, Huissoud C, Kocot A, et al. Non-invasive high-intensity focused ultrasound treatment of the placenta: a preliminary in-vivo study using a simian model. Ultrasound Obstet Gynecol 2017;50:635-41.

18. Sebire NJ, Souka A, Skentou H, et al. Early prediction of severe twin-to-twin transfusion syndrome. Hum Reprod 2000;15:2008-10.

19. D'Antonio F, Khalil A, Dias T, et al. Crown-rump length discordance and adverse perinatal outcome in twins: analysis of the Southwest Thames Obstetric Research Collaborative (STORK) multiple pregnancy cohort. Ultrasound Obstet Gynecol 2013;41:621-6.

20. Wohlmuth C, Osei FA, Moise KJ Jr, et al. Changes in ductus venosus flow profile in twin-twin transfusion syndrome: role in risk stratification. Ultrasound Obstet Gynecol 2016;48:744-51.

(English Language Editor: J. Jones) 